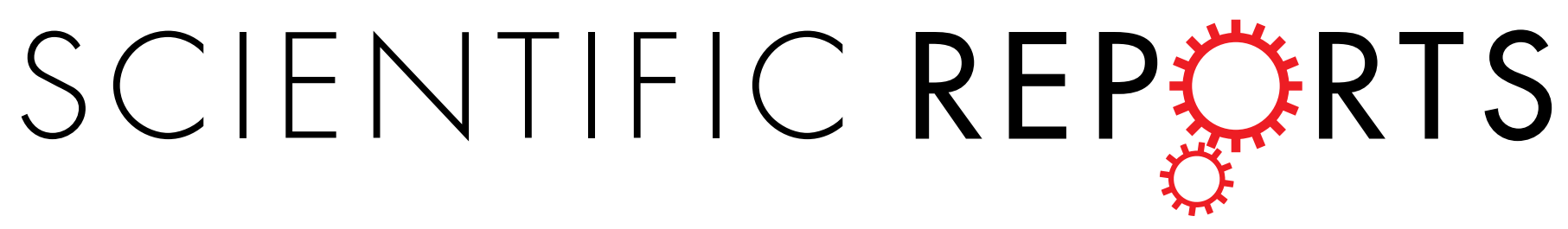

OPEN

Published online: 02 August 2018

\title{
Author Correction: CREB-binding protein plays key roles in juvenile hormone action in the red flour beetle, Tribolium Castaneum
}

Jingjing X $u^{1}$, Amit Roy $\mathbb{( i D}^{1,2}$ \& Subba Reddy Palli $\mathbb{1}^{1}$

Correction to: Scientific Reports https://doi.org/10.1038/s41598-018-19667-6, published online 23 January 2018

This Article contains errors in the primer sequences reported in Table 1. The correct Table 1 appears below.
${ }^{1}$ Department of Entomology, College of Agriculture, Food and Environment University of Kentucky, Lexington, KY, 40546, USA. ${ }^{2}$ Faculty of Forestry and Wood Sciences, EXTEMIT-K, Czech University of Life Sciences, Kamýcká 1176, Prague 6, Suchdol, 165 21, Czech Republic. Correspondence and requests for materials should be addressed to S.R.P. (email: rpalli@uky.edu) 


\begin{tabular}{|c|c|c|}
\hline Gene Name & & Primer sequence $\left(5^{\prime}-3^{\prime}\right)$ \\
\hline \multicolumn{3}{|c|}{ qRT-PCR primers } \\
\hline \multirow{2}{*}{ HSP90 } & $\mathrm{F}$ & GCGCTAAGTGAAGAGCTAAGA \\
\hline & $\mathrm{R}$ & ATGCACACACGAACAAATCAC \\
\hline \multirow{2}{*}{$4 \mathrm{EBP}$} & $\mathrm{F}$ & ATCACCGATGGCAAGACAAGTGAC \\
\hline & $\mathrm{R}$ & ATGGCAGTTCAGAAGGGTCGTTGA \\
\hline \multirow{2}{*}{ СBР } & F & GGTCCCGATGGTAAGAAGAAAG \\
\hline & $\mathrm{R}$ & CCGAGAGATCATTACCCGTTTG \\
\hline \multirow{2}{*}{ G13402 } & $\mathrm{F}$ & ACTGTGCCGAGTTTAGGA \\
\hline & $\mathrm{R}$ & GAATGCCAGTGGGTCAGG \\
\hline \multirow{2}{*}{ HR4 } & $\mathrm{F}$ & ATAGAACAGCTCCGGCAAGA \\
\hline & $\mathrm{R}$ & TGCCGCAGATTGTATCTCTG \\
\hline \multirow{2}{*}{ E75A } & F & GAAATCGCGTCCAAGTG \\
\hline & $\mathrm{R}$ & GAAGGAAGTTCAATGGC \\
\hline \multirow{2}{*}{ E75B } & $\mathrm{F}$ & ATGCAGACCGCCACCATCG \\
\hline & $\mathrm{R}$ & CGGGGATGGAGCTGGAGG \\
\hline \multirow{2}{*}{ E74 } & F & GCGTCTTCAAGCTGGTG \\
\hline & $\mathrm{R}$ & CAGCCTTTGACCGTCCAC \\
\hline \multirow{2}{*}{ FTZ-F1 } & $\mathrm{F}$ & TGCGAGGAATCACAAACAAG \\
\hline & $\mathrm{R}$ & TGTGACGTTTGCTCGAAGAC \\
\hline \multirow{2}{*}{ Rp49 } & F & TGACCGTTATGGCAAACTCA \\
\hline & $\mathrm{R}$ & TAGCATGTGCTTCGTTTTGG \\
\hline \multicolumn{3}{|c|}{ dsRNA primers } \\
\hline \multirow{2}{*}{ СВР } & $\mathrm{F}$ & CCTCAGACTCCTCAGCTTACTA \\
\hline & $\mathrm{R}$ & GAGCTGAAGTTGGCGTAAATTG \\
\hline \multicolumn{3}{|l|}{ Chip Assay } \\
\hline \multirow{2}{*}{$\mathrm{Kr}-\mathrm{h} 1$} & F & GGCTGCAGACGACTTTCTTTA \\
\hline & $\mathrm{R}$ & GCCGGAAATGGTCGGTTATTA \\
\hline \multirow{2}{*}{ G13402 } & $\mathrm{F}$ & GACTGTCATCCACAGGGAAA \\
\hline & $\mathrm{R}$ & CAAGGTGTCCTGCAACATTATC \\
\hline \multirow{2}{*}{$4 \mathrm{EBP}$} & $\mathrm{F}$ & ACTGCCGATGCAAGTCAA \\
\hline & $\mathrm{R}$ & CAGTACGAACGGAGACCATAAC \\
\hline \multirow{2}{*}{ HR4 } & $\mathrm{F}$ & AACGATAACCTTGGGCGATTA \\
\hline & $\mathrm{R}$ & CGATCATAATTTGGACGCTCTTG \\
\hline \multirow{2}{*}{ hsp90 } & F & GCGCTAAGTGAAGAGCTAAGA \\
\hline & $\mathrm{R}$ & ATGCACACACGAACAAATCAC \\
\hline
\end{tabular}

Table 1. Primers used in the paper.

Open Access This article is licensed under a Creative Commons Attribution 4.0 International License, which permits use, sharing, adaptation, distribution and reproduction in any medium or format, as long as you give appropriate credit to the original author(s) and the source, provide a link to the Creative Commons license, and indicate if changes were made. The images or other third party material in this article are included in the article's Creative Commons license, unless indicated otherwise in a credit line to the material. If material is not included in the article's Creative Commons license and your intended use is not permitted by statutory regulation or exceeds the permitted use, you will need to obtain permission directly from the copyright holder. To view a copy of this license, visit http://creativecommons.org/licenses/by/4.0/.

(C) The Author(s) 2018 